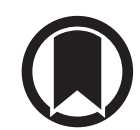

CrossMark

\section{Antinuclear antibodies and subclinical interstitial lung disease in community-dwelling adults: the MESA study}

To the Editor:

The presence of a systemic autoimmune rheumatic disease (ARD) is a well-known risk factor for interstitial lung disease (ILD). For example, $33 \%$ of adults with rheumatoid arthritis (RA) have subclinical ILD [1]. Higher serum levels of IgM rheumatoid factor (RF), IgA RF, and anti-cyclic citrullinated peptide antibody 2 are associated with subclinical ILD in community-dwelling adults [2]. It is unknown whether this relationship between autoimmunity and subclinical ILD is limited to RA-related autoantibodies, or extends more broadly to other epitopes. High attenuation areas (HAA) and interstitial lung abnormalities (ILA) are validated quantitative and qualitative subclinical ILD phenotypes, respectively. In community-dwelling adults, greater HAA is associated with reduced forced vital capacity, reduced exercise capacity, elevated serum levels of matrix metalloproteinase-7 and interleukin-6, higher prevalence of ILA on computed tomography (CT) scans of the chest, higher all-cause mortality rate, and an increased risk of developing clinically evident ILD and ILD-specific mortality at 12-year follow-up [3, 4]. ILA has been associated with all-cause mortality in four different longitudinal cohorts [5]. The purpose of this study was to examine the association between antinuclear antibody (ANA) and both HAA and ILA in community-dwelling adults enrolled in the Multi-Ethnic Study of Atherosclerosis (MESA).

MESA is a population-based cohort study of 6814 adults aged 45-84 years when they were enrolled from 2000 to 2002 without regard to lung disease or ARD [6]. Cardiac CT scans were performed in 6812 participants at examination 1 (2000-2002) [7] and full lung CT scans in 2907 participants at examination 5 (2010-2012). Measurement of HAA, defined as the percentage of lung volume with attenuation values between -600 and -250 Hounsfield units, and ILA, defined as ground glass abnormalities, reticular abnormalities, diffuse centrilobular nodularity, nonemphysematous cysts, honeycombing, and traction bronchiectasis affecting $>5 \%$ of a lung zone in a non-dependent manner [8], has been previously described [3]. HAA was quantified on the 6812 examination 1 cardiac CT scans. Each of the 2907 examination 5 full lung CT scans was visually inspected by one expert radiologist for the presence or absence of ILA. ANA was measured in frozen examiniation 1 sera from 6626 participants using indirect immunofluorescence with HEp-2 cell substrate at TheraTest Labs (TheraTest Labs Inc, Lombard, IL, USA) [9]. Intra-assay coefficient of variation was $<10 \%$ [9]. ANA level was expressed in units. An ANA value $>10$ units was defined as positive. We examined the linearity of the associations between ANA and both HAA and ILA using generalised additive models with loess smoothing functions. We used multiple linear regression to examine associations between natural log-transformed ANA and natural log-transformed HAA, controlling for age, sex, race/ethnicity, body mass index (BMI), height, waist circumference, pack-years of smoking, current smoking status, estimated glomerular filtration rate, study site, education, total imaged lung volume, percent emphysema, and tube current. To ease interpretation of our beta coefficients of natural log-transformed ANA, we have presented base 2 exponentiated beta coefficients, which are the percent differences in HAA per doubling of ANA. We estimated prevalence ratios (PR) for the associations between $\log _{2}$-transformed ANA and ILA using Poisson regression with robust standard error estimation, controlling for age, sex, race/ethnicity, pack-years of smoking, and current smoking status. We performed analyses stratified by age, sex, race/ethnicity, smoking status and BMI. We used likelihood ratio

@ERSpublications

Antinuclear antibodies are associated with subclinical interstitial lung disease in community-dwelling adults enrolled in the Multi-Ethnic Study of Atherosclerosis http://bit.ly/2FIEJpS

Cite this article as: Bernstein EJ, Austin JHM, Kawut SM, et al. Antinuclear antibodies and subclinical interstitial lung disease in community-dwelling adults: the MESA study. Eur Respir J 2020; 55: 1902262 [https://doi.org/10.1183/13993003.02262-2019]. 



FIGURE 1 a) Continuous relationship of antinuclear antibody at examination 1 with predicted (adjusted) percent high attenuation areas at examination $1(\mathrm{n}=6626)$. Smoothed regression line (solid black line) is adjusted for age, sex, race/ethnicity, body mass index, height, waist circumference, pack-years of smoking, current smoking status, estimated glomerular filtration rate, study site, education, total imaged lung volume, percent emphysema, and tube current. Dotted lines are the $95 \%$ confidence bands. Each vertical tick mark on the rug plot along the internal border of the $x$-axis represents one study participant. Overall $p$-value for association $<0.001, p$-value for non-linearity 0.002 . b) Continuous relationship of antinuclear antibody at examination 1 with the predicted probability of interstitial lung abnormalities at examination 5 ( $n=2366$ ). Smoothed regression lines (black solid line: age 45-59 years at examination 1; red dashed line: age 60-84 years at examination 1) are adjusted for age, sex, race/ethnicity, pack-years of smoking, and current smoking status. Dotted lines are the $95 \%$ confidence bands. Each vertical tick mark on the rug plot along the internal border of the $x$-axis represents one study participant. In participants aged $45-59$ years ( $n=1256$ ), overall $p$-value for association $0.009, p$-value for non-linearity 0.58 . In participants aged $60-84$ years $(n=1110)$, overall $p$-value for association 0.68 , $p$-value for non-linearity 0.12 .

tests to test for effect modification, and multiple imputation by chained equations to account for missing covariate data [10]. Only $0.4 \%$ of participants had any missing data. Analyses were performed in STATA, version 15.1 (College Station, TX, USA) and R, version 3.6.1 (R Foundation for Statistical Computing, Vienna, Austria).

The baseline characteristics of the MESA cohort have been previously published [3]. Of the 2430 participants with non-equivocal measurements of ILA at examination 5, ANA was measured in 2366 at examination 1. Thus, 6626 and 2366 participants were included in the HAA and ILA analyses, respectively. Mean age at examination 1 was $62 \pm 10$ years; 53\% (3516/6626) were female. Of the 6626 participants included in the HAA analyses, 39\% identified themselves as white, $27 \%$ as African American, $12 \%$ as Chinese American, and $22 \%$ as Hispanic. $41 \%$ (2688/6623) were former smokers; $14 \%(927 / 6623)$ were current smokers. $11 \%(741 / 6626)$ were ANA positive. Median ANA was 4 units (interquartile range (IQR) 3-7). Median HAA was 5.62\% (IQR 4.55-7.19\%). ILA prevalence was $12.4 \%(293 / 2366)$.

In an unadjusted model, HAA at examination 1 increased by $3.50 \%$ (95\% CI 2.25 to $4.77 \%$; p $<0.001)$ per doubling of ANA at examination 1. In a fully adjusted model, HAA increased by $1.83 \%$ (95\% CI 1.12 to 2.55\%; $\mathrm{p}<0.001$ ) per doubling of ANA (figure 1a). In a fully adjusted model, the p-value for the interaction between ANA and race/ethnicity was 0.04 . In fully adjusted models, HAA increased by $2.82 \%$ (95\% CI 1.49 to $4.16 \%$; $\mathrm{p}<0.001$ ) among African Americans, $2.93 \%$ (95\% CI 0.85 to $5.06 \%$; $\mathrm{p}=0.006$ ) among Chinese Americans, and $2.14 \%$ (95\% CI 0.65 to $3.66 \%$; $=0.005$ ) among Hispanic subjects per doubling of ANA. There was no statistically significant association between ANA and HAA among white subjects $(0.65 \%$ increase per doubling of ANA, $95 \%$ CI -0.46 to $1.76 \% ; \mathrm{p}=0.25)$. There was no statistically significant interaction between ANA and age, sex, smoking status or BMI ( $p$-value for interaction $>0.50$ for each).

In an unadjusted model, the prevalence of ILA at examination 5 increased by $17 \%$ per doubling of ANA at examination 1 (PR 1.17, 95\% CI 1.04-1.33; p=0.01). However, in a fully adjusted model, there was no statistically significant association between ANA and ILA prevalence (PR 1.07, 95\% CI 0.94-1.22; $\mathrm{p}=0.29$ ). In a fully adjusted model, the p-value for the interaction between ANA and age was 0.003 . In a fully adjusted model, ILA increased by 33\% per doubling of ANA (PR 1.33, 95\% CI 1.09-1.63; p=0.006) among younger participants (age 45-59 years at examination 1) (figure 1b). There was no statistically significant association between ANA and ILA prevalence among older participants (age 60-84 years at examination 1) $(\mathrm{PR} 0.97,95 \%$ CI $0.83-1.13$, p-value $=0.68)$. There was no statistically significant interaction between ANA and sex, race/ethnicity, or smoking status ( $\mathrm{p}$-value for interaction $>0.10$ for each). 
In this study, we demonstrated a positive association between levels of ANA, a marker of autoimmunity, and HAA, a quantitative CT biomarker of subclinical ILD. The association between ANA and HAA was strongest among non-white participants and the association between ANA and ILA was stronger among younger participants. Our results indicate that the relationship between autoimmunity and subclinical ILD is not limited to RA-related autoantibodies, but includes other epitopes as well.

It is well-established that autoantibodies precede the development of clinical manifestations of ARDs by several years $[11,12]$. ANA was detected in the serum of $77 \%(89 / 115)$ of military personnel with systemic lupus erythematosus (SLE) [11] and in the serum of 44\% (23/44) of Swedish patients with Sjogren's syndrome prior to symptom onset [12]. Our findings suggest that higher serum ANA levels may also be a risk factor for subclinical ILD.

It is perhaps not surprising that race/ethnicity and age modified the effect of ANA on HAA and ILA, respectively. The prevalence of ANA is modestly higher among African Americans than among non-Hispanic whites in the USA [13], and the prevalence of certain ARDs is higher in non-white populations. For example, African Americans, Asian Americans, and Hispanic Americans have a higher prevalence of SLE than do white subjects in the USA [14]. Moreover, non-white populations tend to have more severe manifestations of ARDs. Among patients with systemic sclerosis, African Americans have a higher prevalence of ILD and more severe ILD than do whites [15]. Although the prevalence of ANA increases with age [13], ARDs such as SLE often present at younger ages [14].

Our study has some limitations. It was cross-sectional, which limits our ability to make causal inferences. Because it was observational, our results may be confounded by unmeasured or poorly measured potential confounders. However, data in MESA were measured with great precision, and we controlled for potential confounders using multivariate modelling approaches to minimise residual confounding.

In summary, our findings provide additional support for the relationship between autoimmunity and subclinical ILD. They suggest autoimmunity may play a role in the pathogenesis of subclinical ILD, even among individuals without an established ARD. Future studies should characterise the specific autoantibody profiles associated with subclinical ILD.

Elana J. Bernstein $\oplus^{1}$, John H.M. Austin ${ }^{2}$, Steven M. Kawut ${ }^{3}$, Ganesh Raghu ${ }^{4}$, Eric A. Hoffman ${ }^{5}$, John D. Newell ${ }^{5}$, Jubal R. Watts, Jr ${ }^{6}$, P. Hrudaya Nath ${ }^{6}$, Sushilkumar K. Sonavane ${ }^{7}$, R. Graham Barr ${ }^{1,8}$ and David J. Lederer ${ }^{1,9}$

${ }^{1}$ Dept of Medicine, Columbia University Irving Medical Center, New York, NY, USA. ${ }^{2}$ Dept of Radiology, Columbia University Irving Medical Center, New York, NY, USA. ${ }^{3}$ Dept of Medicine and Center for Epidemiology and Biostatistics, University of Pennsylvania Perelman School of Medicine, Philadelphia, PA, USA. ${ }^{4}$ Dept of Medicine, University of Washington, Seattle, WA, USA. ${ }^{5}$ Dept of Radiology, University of Iowa Carver College of Medicine, Iowa City, IA, USA. ${ }^{6}$ Dept of Radiology, University of Alabama at Birmingham School of Medicine, Birmingham, AL, USA. ${ }^{7}$ Dept of Radiology, Mayo Clinic, Jacksonville, FL, USA. ${ }^{8}$ Dept of Epidemiology, Columbia University Irving Medical Center, New York, NY, USA. ${ }^{9}$ Regeneron Pharmaceuticals, Inc., Tarrytown, NY, USA.

Correspondence: Elana J. Bernstein, Columbia University Irving Medical Center, 630 West 168th Street, Suite 3-450, New York, NY 10032, USA. E-mail: ejb2153@cumc.columbia.edu

Received: 27 Nov 2019 | Accepted after revision: 23 Dec 2019

Acknowledgements: The authors thank the other investigators, the staff, and the participants of the MESA study for their valuable contributions. A full list of participating MESA investigators and institutions can be found at www. mesa-nhlbi.org

Support statement: This research was supported by contracts HHSN268201500003I, N01-HC-95159, N01-HC-95160, N01-HC-95161, N01-HC-95162, N01-HC-95163, N01-HC-95164, N01-HC-95165, N01-HC-95166, N01-HC-95167, N01-HC-95168 and N01-HC-95169 from the National Heart, Lung, and Blood Institute, and by grants UL1-TR-000040, UL1-TR-001079, and UL1-TR-001420 from the National Center for Advancing Translational Sciences (NCATS). E.J. Bernstein's work was supported by K23-AR-075112. Funding information for this article has been deposited with the Crossref Funder Registry.

Conflict of interest: E.J. Bernstein reports grants from NIH/NIAMS, grants and personal fees from Boehringer Ingelheim, grants from Pfizer, outside the submitted work. J.H.M. Austin has nothing to disclose. S.M. Kawut reports grants from NIH, non-financial support for travel from ATS, and grants from Actelion, United Therapeutics, Gilead, Lung Biotech, Bayer, and Mallinkrodt to the Perelman School of Medicine for CME courses; grants and non-financial support from Cardiovascular Medical Research and Education Fund and non-financial support from Pulmonary Hypertension Association; and has served in an advisory capacity (for grant review and other purposes) for United Therapeutics, Akros Pharmaceuticals, GlaxoSmithKline, and Complexa, Inc. without financial support or in-kind benefits. G. Raghu reports grants and personal fees from Boehringer Ingelheim, outside the submitted work. E.A. Hoffman reports grants from NIH, during the conduct of the study; and is a founder and shareholder of VIDA Diagnostics, a company commercialising lung image analysis software developed, in part, at the University of Iowa. J.D. Newell reports grants from NIH, during the conduct of the study; personal fees as medical advisor from VIDA, grants from NIH and Siemens Healthineers, outside the submitted work; and has patents with VIDA and University of 
Iowa issued. J.R. Watts reports personal fees for lectures from Genentech, Boehringer Ingelheim and France Foundation, outside the submitted work. P.H. Nath has nothing to disclose. S.K. Sonavane reports grants from NIH, during the conduct of the study. R.G. Barr reports grants from NIH, during the conduct of the study; grants from NIH and COPD Foundation, outside the submitted work. D.J. Lederer is a full time employee of Regeneron Pharmaceuticals; the work in this article was performed solely while D.J. Lederer was an employee of Columbia University and does not represent work by Regeneron Pharmaceuticals, Inc.; D.J. Lederer reports personal fees from Roche, Sanofi Genzyme, Philips Respironics, Fibrogen, Global Blood Therapeutics, Boehringer-Ingelheim, Veracyte, and Galapagos unrelated to the current work; institutional grant support from Fibrogen, Global Blood Therapeutics and Boehringer Ingelheim; has performed unpaid consulting work for Galecto, Pliant Therapeutics and Bristol Myers Squibb; Columbia University has received fees from the Pulmonary Fibrosis Foundation for consulting services provided by D.J. Lederer.

\section{References}

1 Gochuico BR, Avila NA, Chow CK, et al. Progressive preclinical interstitial lung disease in rheumatoid arthritis. Arch Intern Med 2008; 168: 159-166.

2 Bernstein EJ, Barr RG, Austin JHM, et al. Rheumatoid arthritis-associated autoantibodies and subclinical interstitial lung disease: the Multi-Ethnic Study of Atherosclerosis. Thorax 2016; 71: 1082-1090.

3 Podolanczuk AJ, Oelsner EC, Barr RG, et al. High attenuation areas on chest computed tomography in community-dwelling adults: the MESA study. Eur Respir J 2016; 48: 1442-1452.

4 Podolanczuk AJ, Oelsner EC, Barr RG, et al. High-attenuation areas on chest computed tomography and clinical respiratory outcomes in community-dwelling adults. Am J Respir Crit Care Med 2017; 196: 1434-1442.

5 Putman RK, Hatabu H, Araki T, et al. Evaluation of CLtIPSEI, Investigators CO. Association Between Interstitial Lung Abnormalities and All-Cause Mortality. JAMA 2016; 315: 672-681.

6 Bild DE, Bluemke DA, Burke GL, et al. Multi-ethnic study of atherosclerosis: objectives and design. Am J Epidemiol 2002; 156: 871-881.

7 Hoffman EA, Jiang R, Baumhauer $\mathrm{H}$, et al. Reproducibility and validity of lung density measures from cardiac CT scans - The Multi-Ethnic Study of Atherosclerosis (MESA) Lung Study. Acad Radiol 2009; 16: 689-699.

8 Washko GR, Hunninghake GM, Fernandez IE, et al. Lung volumes and emphysema in smokers with interstitial lung abnormalities. N Eng J Med 2011; 364: 897-906.

9 TheraTest Laboratories, Inc. EL-ANAscrTM: An Enzyme Immunoassay for the Screening of Human Serum to Detect Antinuclear Antibodies (ANAs). https://docs.wixstatic.com/ugd/c475d7_48e10d5103ec47138e7a182f2650f d15.pdf Date last accessed: 4 April, 2019.

10 Rubin DB, Schenker N. Multiple imputation in health-care databases: an overview and some applications. Stat Med 1991; 10: 585-598.

11 Arbuckle MR, McClain MT, Rubertone MV, et al. Development of autoantibodies before the clinical onset of systemic lupus erythematosus. N Eng J Med 2003; 349: 1526-1533.

12 Jonsson R, Theander E, Sjostrom B, et al. Autoantibodies present before symptom onset in primary Sjogren syndrome. JAMA 2013; 310: 1854-1855.

13 Satoh M, Chan EK, Ho LA, et al. Prevalence and sociodemographic correlates of antinuclear antibodies in the United States. Arthritis Rheum 2012; 64: 2319-2327.

14 Petri M. Epidemiology of systemic lupus erythematosus. Best Pract Res Clin Rheumatol 2002; 16: 847-858.

15 Steen V, Domsic RT, Lucas M, et al. A clinical and serologic comparison of African American and Caucasian patients with systemic sclerosis. Arthritis Rheum 2012; 64: 2986-2994. 